\title{
Equation of state of nuclear matter from empirical constraints
}

\author{
N. Alam, B. K. Agrawal, J. N. De, and S. K. Samaddar \\ Saha Institute of Nuclear Physics, 1/AF Bidhannagar, Kolkata 700064, India
}

G. Colò

\begin{abstract}
Dipartimento di Fisica, Università degli Studi di Milano, via Celoria 16, I-20133 Milano, Italy and INFN, sezione di Milano, via Celoria 16, I-20133 Milano, Italy
\end{abstract}

(Received 16 April 2014; revised manuscript received 14 October 2014; published 14 November 2014)

\begin{abstract}
From empirically determined values of some of the characteristic constants associated with homogeneous nuclear matter at saturation and subsaturation densities, within the framework of a Skyrme-inspired energy density functional, we construct an equation of state $(\mathrm{EoS})$ of nuclear matter.This EoS is then used to predict values of density slope parameters of symmetry energy $L(\rho)$, isoscalar incompressibility $K(\rho)$, and a few related quantities. The close consonance of our predicted values with the currently available ones for the density dependence of symmetry energy and incompressibility gleaned from diverse approaches offers the possibility that our method may help in settling their values in tighter bounds. Extrapolation of our EoS at supranormal densities shows that it is in good harmony with the one extracted from experimental data.
\end{abstract}

DOI: 10.1103/PhysRevC.90.054317

PACS number(s): 21.65.Mn, 21.65.Ef, 21.10.Gv, 26.60.-c

\section{INTRODUCTION}

The nuclear equation of state (EoS) entails in a broad sweep knowledge of the diverse properties of nuclear matter: its saturation density, energy per nucleon $e(\rho)$ ( $\rho$ is the density), incompressibility $K(\rho)$, the symmetry energy and its density content, i.e., the symmetry coefficient $e_{\text {sym }}(\rho)$, the symmetry slope parameter $L(\rho)$, the symmetry incompressibility $K_{\tau}(\rho)$, and all the higher symmetry derivatives. Attention has been naturally drawn in recent times toward having a refined understanding of this nuclear EoS from both experimental and theoretical sides. The binding energies of stable atomic nuclei are the most accurately known experimental entities in nuclear physics; these supplemented with knowledge of giant monopole and dipole resonances followed by theoretical analysis have yielded some of the EoS parameters such as the saturation density $\rho_{0}$ of symmetric nuclear matter and $e\left(\rho_{0}\right), K\left(\rho_{0}\right)$, and $e_{\text {sym }}\left(\rho_{0}\right)$ in reasonably tight bounds [1-6]. The knowledge of the symmetry derivatives $L\left(\rho_{0}\right)$ and $K_{\tau}\left(\rho_{0}\right)$ is still not very certain [1,7-9]. Analyses of the different nuclear observables do not help much in removing the uncertainty. Correlation systematics of nuclear isospin with neutron skin thickness [10,11], isospin diffusion [12,13], nucleon emission ratios [14], or isoscaling [15] in heavy ion collisions, all yield values of the symmetry slope parameter $L_{0}\left[=L\left(\rho_{0}\right)\right]$ that are not much in consonance with one another. An attempt was recently made to constrain $L_{0}$ in tighter bounds from nuclear masses aided by microscopic calculations $[16,17]$ on the neutron skin of heavy nuclei; it was found to give $L_{0}=59 \pm 13.0 \mathrm{MeV}$. Other recent attempts, from analysis of the isovector giant dipole and quadrupole resonances in the ${ }^{208} \mathrm{~Pb}$ nucleus $[18,19]$ give $L_{0}=$ $43 \pm 26$ and $37 \pm 18 \mathrm{MeV}$ respectively. This underscores the still unresolved uncertainty in getting to the value of $L_{0}$ and asks for newer avenues to understand it. The present state of the art on symmetry energy and related parameters can be found in the topical issue on nuclear symmetry energy [20].
The EoS parameters so mentioned pertain to only one density: the saturation density $\rho_{0}$. If all of them are known precisely, it is in principle possible to construct with a suitable energy density functional (EDF) the nuclear $\operatorname{EoS} e(\rho, \delta)$ where $\delta=\left(\rho_{n}-\rho_{p}\right) /\left(\rho_{n}+\rho_{p}\right)$ is the isospin asymmetry. Whereas the high density end of the EoS would be of immediate value in understanding the dynamical evolution of the core collapse of a massive star and the associated explosive nucleosynthesis [21,22], or the radii and lower bound of the maximum mass of cold neutron stars [23], the low-density end helps in getting a closer estimate of the neutron skin thickness or the neutron density distribution $[10,11,16,17]$ in neutronrich nuclei. Accurate knowledge about the EoS parameters at densities other than $\rho_{0}$ may put the nuclear EoS on firmer ground; unfortunately, they are very scanty. At density higher than $\rho_{0}$, information from experimental data has still large uncertainty [24]; at subsaturation density, from giant dipole resonance analysis, a quantitative constraint on $e_{\text {sym }}(\rho)$ could be put as $23.3<e_{\text {sym }}\left(\rho=0.1 \mathrm{fm}^{-3}\right)<24.9 \mathrm{MeV}$ [7]. Further information derived from theoretical analyses at around this density may be of added significance: (i) the energy per nucleon of neutron matter is $\sim 10.9 \pm 0.5 \mathrm{MeV}$ at $\rho=0.1$ $\mathrm{fm}^{-3}$ [25] and (ii) the density derivative of the nuclear incompressibility $M_{c}=3 \rho d K /\left.d \rho\right|_{\rho=\rho_{c}} \simeq 1100 \pm 70 \mathrm{MeV}$ where $\rho_{c}$ is $\simeq 0.7 \rho_{0}$ [26].

As is evident from the previous discussion, the plethora of nuclear EoS failed to effectively constrain the density content of the nuclear symmetry energy and the nuclear incompressibility from fits to diverse microscopic nuclear data. The reason lies in the choice of different sets of microscopic observables to be fitted. The isoscalar and isovector quantities associated with nuclear matter, however, have emerged to be very well constrained. The isoscalar entities are (i) $e_{0}\left[=e\left(\rho_{0}\right)=-16.0 \pm 0.1 \mathrm{MeV}\right]$, (ii) saturation density $\rho_{0}\left(=0.155 \pm 0.008 \mathrm{fm}^{-3}\right)$, where the pressure $P\left(\rho_{0}\right)=0$, and (iii) the incompressibility coefficient $K_{0}\left[=K\left(\rho_{0}\right)=\right.$ $9 \partial^{2} e /\left.\partial \rho^{2}\right|_{\rho_{0}}=9 d P /\left.d \rho\right|_{\rho_{0}}=240 \pm 20 \mathrm{MeV}$ ] [27]. All these 
quantities refer to symmetric nuclear matter. The isovector quantities are (iv) $e_{\text {sym }}\left(\rho_{0}\right)(=32.1 \pm 0.31 \mathrm{MeV})[4]$ and $(\mathrm{v})$ $e_{\text {sym }}\left(\rho=0.1 \mathrm{fm}^{-3}\right)(=24.1 \pm 0.8 \mathrm{MeV})[7]$.

In this article, we have tried to find how the input of the empirical knowledge of these quantities can be used to construct an EDF for nuclear matter and to predict the as yet not so well-constrained density dependence of its symmetry properties in reasonably tighter bounds. These isoscalar and isovector nuclear parameters effectively contain condensed experimental information on the bulk nuclear properties. As opposed to direct investigation of the microscopic properties of nuclei as done, e.g., in Ref. [28], which can lead to somewhat different predictions depending on the observables chosen to be explored, the alternate edifice for the nuclear EDF built in this article on the well established nuclear bulk parameters is so structured, as we see later, that it gives an easy and transparent look at the correlations of the predicted values of the density derivatives of the symmetry energy and the nuclear incompressibility to the input parameters. Furthermore, if the values of the density derivatives $L_{0}, K_{\tau}, M_{0}$, etc. could be well settled by as yet other unexplored means and differ from our predicted values, the foundation, i.e., the values of the nuclear bulk parameters or the choice of the Skyrme EDF, then become subjects of fresher scrutiny. Computationally, our method is also much less intensive. To our knowledge, a comprehensive study of this kind has not been done before.

Henceforth, the quantities corresponding to the densities $\rho_{0}$ and $\rho=0.1 \mathrm{fm}^{-3}$ will be denoted with the subscripts 0 and 1 , respectively (like $e_{\mathrm{sym}, 0}, e_{\mathrm{sym}, 1}$, etc.). The value of $\rho_{0}$ is an indirectly obtained entity. From acceptable Skyrme energy density functionals, it is $\sim 0.16 \mathrm{fm}^{-3}$ [29] whereas the relativistic mean-field models give a value of $\rho_{0}$ in the vicinity of $\sim 0.15 \mathrm{fm}^{-3}[30,31]$. Our choice for $\rho_{0}$ covers this range.

The paper is organized as follows. Section II contains a brief discussion of the theoretical elements. Results and discussions are presented in Sec. III. Section IV contains the concluding remarks.

\section{THEORETICAL EDIFICE}

The Skyrme framework is chosen for the energy density functional [32]. The energy per nucleon for nuclear matter is then

$$
\begin{aligned}
e(\rho, \delta)= & a_{1}\left[\left(\frac{1+\delta}{2}\right)^{5 / 3}+\left(\frac{1-\delta}{2}\right)^{5 / 3}\right] \rho^{2 / 3} \\
& +\left(b_{1}+b_{2} \delta^{2}\right) \rho+\left(c_{1}+c_{2} \delta^{2}\right) \rho^{\alpha+1} \\
& +\left[d_{1}\left\{\left(\frac{1+\delta}{2}\right)^{5 / 3}+\left(\frac{1-\delta}{2}\right)^{5 / 3}\right\}\right. \\
& \left.+d_{2}\left\{\left(\frac{1+\delta}{2}\right)^{8 / 3}+\left(\frac{1-\delta}{2}\right)^{8 / 3}\right\}\right] \rho^{5 / 3} .
\end{aligned}
$$

The first term on the right-hand side is the free Fermi gas energy, $a_{1}=\frac{\hbar^{2}}{2 m} \frac{3}{5}\left(3 \pi^{2}\right)^{2 / 3}=119.14 \mathrm{MeV} \mathrm{fm}^{2}$, where $m$ is the nucleon mass. For the chosen values of $e_{0}$ and $\rho_{0}$, values of $\alpha$ ranging only from $1 / 6$ to $1 / 3$ allow for an acceptable set of $\left(m^{*} / m, K_{0}\right)$ [33] where $m^{*}$ is the nucleon effective mass. We therefore chose $\alpha=0.2 \pm 0.1$; this allows $m^{*} / m$ to lie in the acceptable range $m^{*} / m \simeq 0.8 \pm 0.2$ [29]. We take the median value of $\alpha=0.2$. We are left with six unknown parameters, namely, $b_{1}, c_{1}, d_{1}, b_{2}, c_{2}$, and $d_{2}$, which would completely define the EDF. As already mentioned, we have, however, five equations, three from isoscalar entities and two from isovector entities.

The isoscalar equations are

$$
\begin{gathered}
e_{0}=\frac{a_{1}}{2^{2 / 3}} \rho_{0}^{2 / 3}+b_{1} \rho_{0}+c_{1} \rho_{0}^{\alpha+1}+\left(\frac{1}{2^{2 / 3}} d_{1}+\frac{1}{2^{5 / 3}} d_{2}\right) \rho_{0}^{5 / 3} \\
P_{0}=0=\rho_{0}^{2}\left[\frac{2}{3} \frac{a_{1}}{2^{2 / 3}} \rho_{0}^{-1 / 3}+b_{1}+c_{1}(\alpha+1) \rho_{0}^{\alpha}\right. \\
\left.+\frac{5}{3}\left(\frac{1}{2^{2 / 3}} d_{1}+\frac{1}{2^{5 / 3}} d_{2}\right) \rho_{0}^{2 / 3}\right]
\end{gathered}
$$

and

$$
\begin{aligned}
K_{0}= & 9\left[\frac{10}{9} \frac{a_{1}}{2^{2 / 3}} \rho_{0}^{2 / 3}+2 b_{1} \rho_{0}+(\alpha+1)(\alpha+2) c_{1} \rho_{0}^{\alpha+1}\right. \\
& \left.+\frac{40}{9}\left(\frac{1}{2^{2 / 3}} d_{1}+\frac{1}{2^{5 / 3}} d_{2}\right) \rho_{0}^{5 / 3}\right] .
\end{aligned}
$$

The isoscalar equations yield the values of $b_{1}, c_{1}$, and $\left(\frac{d_{1}}{2^{2 / 3}}+\right.$ $\left.\frac{d_{2}}{2^{5 / 3}}\right)$. The isovector equations, evaluated at $\rho_{0}$ and $\rho_{1}(=0.1$ $\mathrm{fm}^{-3}$ ) are given by

$$
\begin{aligned}
e_{\text {sym }}(\rho)= & \left.\frac{1}{2} \frac{\partial^{2} e(\rho)}{\partial \delta^{2}}\right|_{\delta=0} \\
= & \frac{5}{9} \frac{a_{1}}{2^{2 / 3} \rho^{2 / 3}+b_{2} \rho+c_{2} \rho^{\alpha+1}} \\
& +\left[\frac{5}{9} \frac{d_{1}}{2^{2 / 3}}+\frac{20}{9} \frac{d_{2}}{2^{5 / 3}}\right] \rho^{5 / 3}
\end{aligned}
$$

To fix the remaining parameters, we need an extra condition in conjunction with the two isovector equations. For this, we take that the energy per particle of isospin asymmetric nuclear matter is quadratic in the asymmetry parameter $\delta$. This condition is found to be an excellent approximation from nearly all energy density functionals [34,35] and also from microscopic calculations in the Bruckner-Hartree-Fock (BHF) formalism [36,37] at all densities up to $\rho_{0}$ and a little beyond. This implies that the difference between the symmetry energy coefficients defined by Eq. (5) and the one by the equation

$$
\begin{aligned}
\tilde{e}_{\mathrm{sym}}(\rho)= & e(\rho, \delta=1)-e(\rho, \delta=0) \\
= & a_{1}\left(1-\frac{1}{2^{2 / 3}}\right) \rho^{2 / 3}+b_{2} \rho+c_{2} \rho^{\alpha+1} \\
& +\left[d_{1}\left(1-\frac{1}{2^{2 / 3}}\right)+d_{2}\left(1-\frac{1}{2^{5 / 3}}\right)\right] \rho^{5 / 3},
\end{aligned}
$$

should be minimal.

To achieve this minimality, we take the help of the equation for the symmetry slope parameter $L$. From its definition 


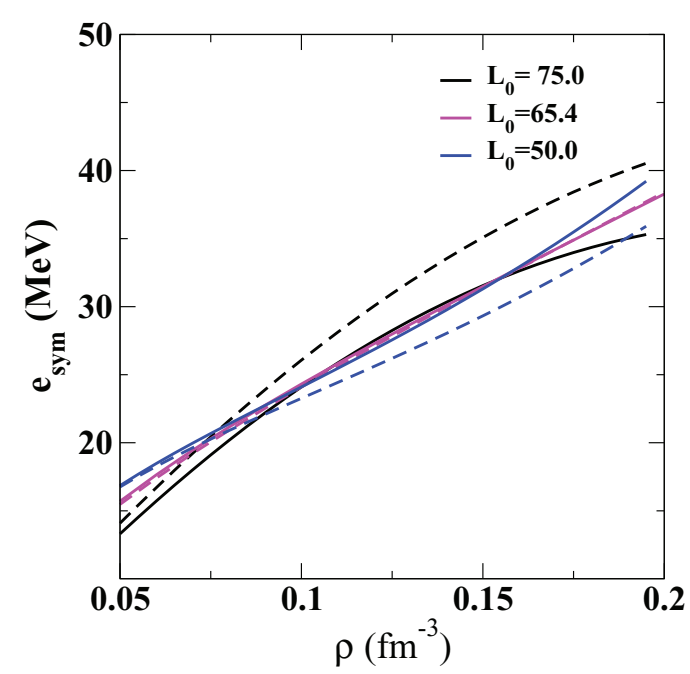

FIG. 1. (Color online) Plots for the variation of $e_{\text {sym }}$ as a function of density $\rho$. The values of $e_{\text {sym }}$ represented by the full and the dashed lines are obtained using two different definitions as given by Eqs. (5) and (6). The blue, magenta, and black lines correspond to $L_{0}=50$, 65.4 , and $75 \mathrm{MeV}$ at $\rho=\rho_{0}$. With $L_{0}=65.4 \mathrm{MeV}$, one magenta line falls over the other; they can-not be distinguished from each other.

$L(\rho)=3 \rho \partial e_{\text {sym }} / \partial \rho$, one gets from Eq. (5)

$$
\begin{aligned}
L(\rho)= & 3 \rho\left[\frac{10}{27} \frac{a_{1}}{2^{2 / 3}} \rho^{-1 / 3}+b_{2}+(\alpha+1) c_{2} \rho^{\alpha}\right. \\
& \left.+\frac{5}{3} \rho^{2 / 3}\left(\frac{5}{9} \frac{d_{1}}{2^{2 / 3}}+\frac{20}{9} \frac{d_{2}}{2^{5 / 3}}\right)\right],
\end{aligned}
$$

With a given value of $L_{0}\left[=L\left(\rho_{0}\right)\right]$, from known $e_{\mathrm{sym}, 0}$ and $e_{\mathrm{sym}, 1}$, at the two densities $\rho_{0}$ and $\rho_{1}$, one can solve for $b_{2}$, $c_{2}$, and $\left[\frac{5}{9} \frac{d_{1}}{2^{2 / 3}}+\frac{20}{9} \frac{d_{2}}{2^{5 / 3}}\right]$. Since $\left(\frac{d_{1}}{2^{2 / 3}}+\frac{d_{2}}{2^{5 / 3}}\right)$ is known from the isoscalar equations, $d_{1}$ and $d_{2}$ are now obtained.

Now that for a given $L_{0}$ all the parameters of the EDF are known, one can calculate $e_{\text {sym }}(\rho)$. With the same set of coefficients $\tilde{e}_{\text {sym }}(\rho)$ can also be calculated. Normally, for an arbitrary value of the given $L_{0}, e_{\mathrm{sym}}$ and $\tilde{e}_{\mathrm{sym}}$ may not be equal; only for a specific value of $L_{0}$ do they tend to be equal (see Fig. 1). To be more specific, as a function of input $L_{0}$, we have calculated the coefficients $b_{1}, b_{2}$, etc. using the relevant equation for $e_{\text {sym }}$ for a set of densities $\rho_{i}$ lying in the range $0.05<\rho<0.2 \mathrm{fm}^{-3}$, calculated $\tilde{e}_{\text {sym }}$ with the same set of coefficients, and have chosen that $L_{0}$ as the requisite one that gives the minimum of $\sum_{i}\left[e_{\mathrm{sym}}\left(\rho_{i}\right)-\tilde{e}_{\mathrm{sym}}\left(\rho_{i}\right)\right]^{2}$. This settles the EoS. In Fig. 1, $e_{\text {sym }}(\rho)$ and $\tilde{e}_{\text {sym }}(\rho)$ are displayed as a function of input $L_{0}$. The full lines refer to $e_{\text {sym }}(\rho)$, the dashed lines to $\tilde{e}_{\text {sym }}(\rho)$. The difference between these two is minimum when $L_{0}=65.4 \mathrm{MeV}$. All the parameters $a_{1}, b_{1}$, $b_{2}$, etc. corresponding to the density functional are listed in Table I.

\section{RESULTS AND DISCUSSIONS}

Once the parameters of the EDF [Eq. (1)] are known, the higher order density derivatives of energy and symmetry energy of nuclear matter may be obtained. They are presented
TABLE I. Parameters of the energy density functional corresponding to the central values of the isoscalar and isovector inputs.

\begin{tabular}{lrcr}
\hline \hline$a_{1}\left(\mathrm{MeV} \mathrm{fm}^{2}\right)$ & 119.14 & $\alpha$ & 0.2 \\
$b_{1}\left(\mathrm{MeV} \mathrm{fm}^{3}\right)$ & -816.95 & $b_{2}\left(\mathrm{MeV} \mathrm{fm}^{3}\right)$ & 744.65 \\
$c_{1}\left(\mathrm{MeV} \mathrm{fm}^{3(\alpha+1)}\right)$ & 724.51 & $c_{2}\left(\mathrm{MeV} \mathrm{fm}^{3(\alpha+1)}\right)$ & -1149.66 \\
$d_{1}\left(\mathrm{MeV} \mathrm{fm}^{5}\right)$ & -32.99 & $d_{2}\left(\mathrm{MeV} \mathrm{fm}^{5}\right)$ & 891.15 \\
\hline \hline
\end{tabular}

in Sec. III A. This EDF can also be used to estimate certain properties of microscopic nuclei like neutron skin thickness of heavy nuclei. This is discussed in Sec. III B. As an aside, the EDF has also been employed to explore some properties of neutron stars, discussion of which is contained in Sec. III C.

\section{A. Nuclear matter: Density derivatives of symmetry energy and isoscalar incompressibility}

Expressions for higher order symmetry derivatives $K_{\mathrm{sym}, 0}$ and $K_{\tau}$ are given by $K_{\mathrm{sym}, 0}=\left.9 \rho_{0}^{2} \frac{\partial^{2} e_{\mathrm{sym}}}{\partial \rho^{2}}\right|_{\rho_{0}}$ and $K_{\tau}=$ $\left.9 \rho_{\delta}^{2} \frac{\partial^{2} e_{\text {sym }}}{\partial \rho^{2}}\right|_{\rho_{\delta}}$. Here $\rho_{\delta}$ is the saturation density of asymmetric nuclear matter corresponding to the asymmetry $\delta$. The symmetry derivatives $K_{\text {sym }, 0}$ and $K_{\tau}$ are related: $K_{\tau}=K_{\text {sym }, 0}-$ $6 L_{0}-\frac{Q_{0} L_{0}}{K_{0}}$, where $Q_{0}=\left.27 \rho_{0}^{3} \frac{\partial^{3} e(\rho, 0)}{\partial \rho^{3}}\right|_{\rho_{0}}$. They all can be evaluated from the EDF parameters. The density derivative of the isoscalar incompressibility $M(\rho)\left(=3 \rho \frac{d K(\rho)}{d \rho}\right)$ of symmetric nuclear matter is also calculated. At the saturation density $\rho_{0}, M_{0}\left(=M\left(\rho_{0}\right)\right)$ equals $12 K_{0}+Q_{0}$. In Table II we list the calculated values of various isoscalar and isovector quantities together with their total uncertainties. The latter are associated with the uncertainties in the six input quantities $Y_{i}\left(=\left\{e_{0}\right.\right.$, $\rho_{0}, K_{0}, e_{\mathrm{sym}, 0}, e_{\mathrm{sym}, 1}$, and $\left.\left.\alpha\right\}\right)$. The extracted value of $L_{0}$ is $65.4 \pm 13.5 \mathrm{MeV}$. It is in excellent consonance with that obtained from analysis of pygmy dipole resonance [38] and in very good agreement with that obtained earlier from nuclear masses and the neutron skin thickness of heavy nuclei $[16,17]$. This is also very consistent with the value $L_{0}=66.5 \mathrm{MeV}$ obtained from a systematic analysis within the BHF approach using a realistic nucleon-nucleon potential [39]. Not much can be said about the reasons behind the good agreement between our present extracted value of $L_{0}$ with that obtained from pigmy dipole resonance [8] except that in this case the value of $e_{\text {sym }}\left(\rho_{0}\right)$ matches extremely well with our input value. The agreement with that obtained from the BHF approach [39] is possibly coincidental; the one aspect that is to be noted here is that all the symmetry derivatives $L_{0}, K_{\text {sym }, 0}$, and $K_{\tau}$ from the BHF approach are in extremely good consonance with our calculated values though the nuclear bulk parameters $\left[\rho_{0}\right.$, $e_{0}, K_{0}$, and $\left.e_{\text {sym }}\left(\rho_{0}\right)\right]$ do not have a good common overlap.

TABLE II. Values of the extracted entities from the nuclear EoS. All quantities are in $\mathrm{MeV}$.

\begin{tabular}{lrcc}
\hline \hline$L_{0}$ & $65.4 \pm 13.5$ & $M_{c}$ & $1150 \pm 91$ \\
$K_{\text {sym }, 0}$ & $-22.9 \pm 73.2$ & $Q_{0}$ & $-344 \pm 56$ \\
$K_{\tau}$ & $-321.6 \pm 34.4$ & $M_{0}$ & $2535 \pm 293$ \\
\hline \hline
\end{tabular}


TABLE III. The observables $X$ are listed in the first column, they are in units of $\mathrm{MeV}$. The second column represents $\Delta X$, the total uncertainty in $X$ from the input uncertainties $\Delta Y_{i}$ in the vector $\mathbf{Y}$ $\left\{e_{0}, \rho_{0}, K_{0}, e_{\mathrm{sym}, 0}, e_{\mathrm{sym}, 1}, \alpha\right\}$. The element $Y_{2}\left(\equiv \rho_{0}\right)$ is in unit of $\mathrm{fm}^{-3}$, $Y_{6}(\equiv \alpha)$ is dimensionless, all other elements in the vector $\mathbf{Y}$ are in units of MeV. The units in the columns $\frac{\partial X}{\partial Y_{i}}(i=1, \ldots 6)$ can then be obtained accordingly.

\begin{tabular}{lrrrcccr}
\hline \hline$X$ & $\Delta X$ & $\frac{\partial X}{\partial Y_{1}}$ & $\frac{\partial X}{\partial Y_{2}}$ & $\frac{\partial X}{\partial Y_{3}}$ & $\frac{\partial X}{\partial Y_{4}}$ & $\frac{\partial X}{\partial Y_{5}}$ & $\frac{\partial}{\partial Y_{6}}$ \\
\hline$L_{0}$ & 13.5 & 0.6 & -1118 & 0.081 & 10.16 & -11.09 & -27.5 \\
$K_{\text {sym }}$ & 73.2 & 12.2 & -3889 & 1.502 & 25.84 & -40.07 & -489 \\
$K_{\tau}$ & 34.4 & -5.4 & 1339 & 0.024 & -20.55 & 10.59 & 309.2 \\
$M_{0}$ & 293 & 54 & -447.5 & 14.6 & 0.00 & 0.00 & -195 \\
$M_{c}$ & 91 & -2.9 & 57.5 & 4.55 & 0.00 & 0.00 & 32.6 \\
$Q_{0}$ & 56 & 54 & -447.5 & 2.6 & 0.00 & 0.00 & -194.9 \\
\hline \hline
\end{tabular}

Most of the uncertainty in our extracted value of $L_{0}$ comes from the uncertainties in the empirically obtained quantities $\rho_{0}$ and $e_{\text {sym }}(\rho)$ at the two densities (see also Table III). If the central value of $\rho_{0}$ is pushed down to $0.14 \mathrm{fm}^{-3}$ keeping all other input parameters same, then the central value of $L_{0}$ shoots up to $86.1 \mathrm{MeV}$. Attention is also drawn to the calculated value of $K_{\tau}$. Analyzing the experimental breathingmode energies of Sn isotopes, Li et al. [40] suggested its value as $-550 \pm 100 \mathrm{MeV}$. This is too strongly negative to be compatible with the behavior of low-density neutron matter [41,42]. Higher order effects such as surface symmetry, present in such analysis in disguise, may have contributed to such a high value. Explicit inclusion of the surface symmetry term seems to lower the value of $K_{\tau}$ to $\sim-350 \mathrm{MeV}[43,44]$. Our present value of $K_{\tau}=-321.6 \pm 34.4 \mathrm{MeV}$ is in very good agreement with this; it is also in close consonance with the value of $-370 \pm 120 \mathrm{MeV}$ extracted from measurements of isospin diffusion in heavy ion collisions. The empirical value of $M_{c}(=1100 \pm 70 \mathrm{MeV})$ obtained from analysis of giant monopole resonance energies of $\mathrm{Sn}$ isotopes and of ${ }^{90} \mathrm{Zr}$ and ${ }^{144} \mathrm{Sm}$ nuclei [26] is very compatible with our calculated value; similarly, the value of $Q_{0}$ calculated by us conforms well with the one $\left(Q_{0}=-350 \pm 30 \mathrm{MeV}\right)$ obtained from examination of a host of standard Skyrme interactions [34].

The total uncertainties in the various quantities considered in Table II are evaluated as [45]

$$
\Delta X=\sqrt{\sum_{i}\left(\Delta X_{i}\right)^{2}},
$$

where $\Delta X_{i}=\frac{\partial X}{\partial Y_{i}} \Delta Y_{i} ; \Delta X$ is the total uncertainty on a given quantity $X$ induced by the associated uncertainties $\Delta Y_{i}$ (= $0.1 \mathrm{MeV}, 0.008 \mathrm{fm}^{-3}, 20 \mathrm{MeV}, 0.31 \mathrm{MeV}, 0.8 \mathrm{MeV}, 0.1$ ) in the input quantities $Y_{i}$. The quantities $\frac{\partial X}{\partial Y_{i}}$ are calculated numerically; their signs reflect the direction of change in $X$ with increase in $Y_{i}$. Table III displays, for the relevant observables $X$, the values of $\frac{\partial X}{\partial Y_{i}}$ along with the associated total uncertainty $\Delta X$. The derivatives $\frac{\partial X}{\partial Y_{i}}$ help in estimating the partial contributions $\Delta X_{i}$ to the total uncertainty $\Delta X$. Once $\frac{\partial X}{\partial Y_{i}}$ are known, it is easy to estimate the change in $\Delta X$ with change in $\Delta Y_{i}$. This table can be an instructive guide in

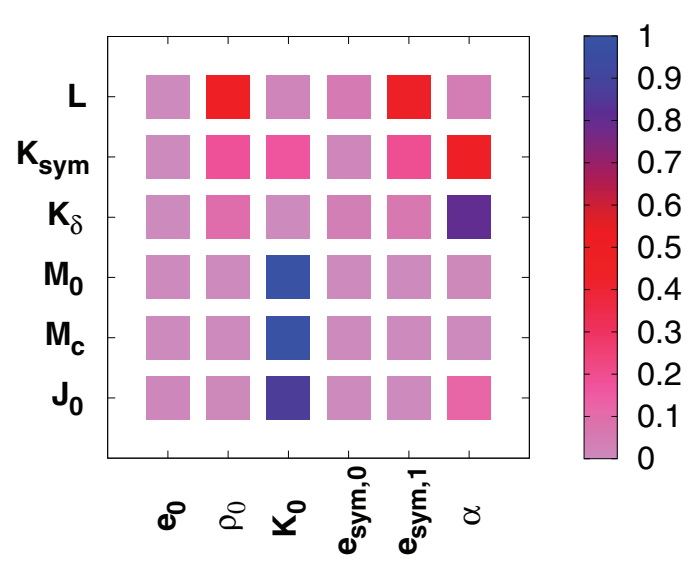

FIG. 2. (Color online) The normalized squared errors, $\left(\frac{\partial X}{\partial Y_{i}} \frac{\Delta Y_{i}}{\Delta X}\right)^{2}$ $\left(=x_{i}^{2}\right)$, are color coded. The quantities $X$ and $Y_{i}$ are given along the ordinate and abscissa respectively.

improving existing energy density functionals as it enables one to understand how the various quantities $X$ can be adjusted by changing $Y_{i}$ 's or vice versa. One may note that the symmetry observables $L_{0}, K_{\mathrm{sym}, 0}$, and $K_{\tau}$ correlate with $e_{\mathrm{sym}, 0}$ and $e_{\mathrm{sym}, 1}$ always in the reverse (see columns 6 and 7 in Table III). This correlated structure of $L_{0}$ on $e_{\mathrm{sym}, 0}$ and $e_{\mathrm{sym}, 1}$ was noticed earlier [46]. Similar correlation of the symmetry observables $K_{\text {sym }, 0}$ and $K_{\tau}$ on $e_{\text {sym }, 0}$ and $e_{\text {sym }, 1}$ is noticed in our calculation.

The fractional contributions $x_{i}^{2}=\left(\frac{\Delta X_{i}}{\Delta X}\right)^{2}=\left(\frac{\partial X}{\partial Y_{i}} \frac{\Delta Y_{i}}{\Delta X}\right)^{2}$ to the uncertainties in the observables $X$ from the uncertainties in the input quantities $Y_{i}$ are shown in Fig. 2 in color code. As one sees, $\sum_{i} x_{i}^{2}=1$. They depict the relative importance of the precision of the input parameters in measuring up the uncertainties in an observable $X$. From the first column in the figure, on can easily see that the uncertainty in energy per particle $e_{0}$ has a negligible role in the uncertainties in the observables $X$ we calculate. One also sees that nearly all the uncertainties in $M_{0}$ and $M_{c}$ emanate from the uncertainty in $K_{0}$ and that the uncertainty in $\alpha$ has a very strong role in the evaluated uncertainty of $K_{\tau}$.

\section{B. Finite nuclei: Neutron skin}

The EoS of infinite homogeneous nuclear matter calculated from the EDF can be beneficially used to estimate some quantities relevant to microscopic nuclear systems. For example, with the calculated values of $L_{0}$ and $K_{\mathrm{sym}, 0}$, one can evaluate $\rho_{A}$, the equivalent density of nuclei, from the equation

$$
e_{\mathrm{sym}}^{s} \simeq A^{1 / 3}\left[L_{0} \epsilon_{A}-\frac{1}{2} K_{\mathrm{sym}, 0} \epsilon_{A}^{2}\right],
$$

where, $e_{\mathrm{sym}}^{s}$ is the surface symmetry energy coefficient. The equivalent density $\rho_{A}$ of a nucleus of mass $A$ is defined as the density at which the symmetry coefficient $e_{\text {sym }}\left(\rho_{A}\right)$ of nuclear matter equals $e_{\text {sym }}(A)$, the symmetry coefficient of the nucleus. The "experimental" value of $e_{\text {sym }}^{s}$ is taken as $58.91 \pm 1.08 \mathrm{MeV}[4] ; \epsilon_{A}=\left(\rho_{0}-\rho_{A}\right) / 3 \rho_{0}$. Figure 3 displays our calculated values of $\rho_{A}$ (shown as a shaded region) as a function of the atomic mass number $A$. The blue triangles in the figure refer to the value of $\rho_{A}$ from Table I of Ref. [10], calculated with different effective interactions for three nuclei, 


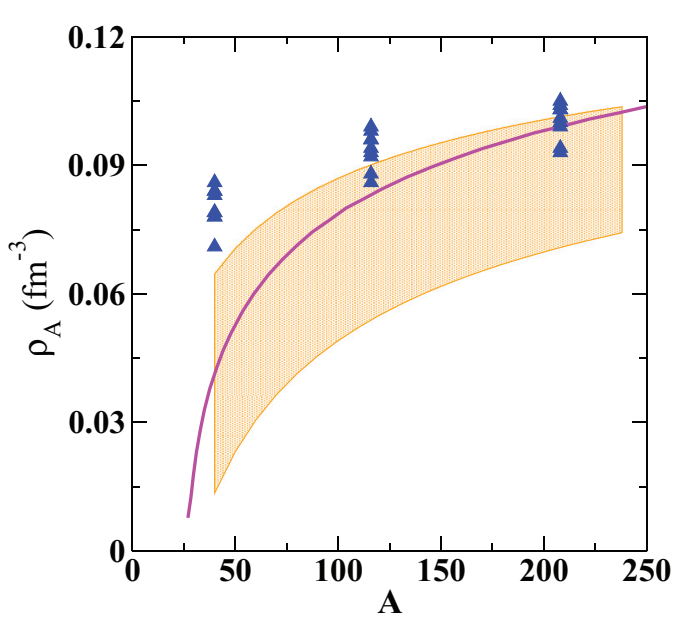

FIG. 3. (Color online) Our results for the equivalent density shown as a shaded region as a function of mass number. The blue triangles are from Ref. [10], the magenta line is from Ref. [6].

$A=40,116$, and 208. The magenta line corresponds to the values calculated in Ref. [6]. Our calculations with the indicated errors are seen to have a good overlap with these results. With known $L_{0}$, an estimate of $r_{\text {skin }}$, the neutron-skin thickness of heavy nuclei, can also be made. For that we make use of the $L_{0}-r_{\text {skin }}$ correlation method as elucidated in Refs. $[10,17]$ for Skyrme interactions. As an example, with our value of $L_{0}$ we obtain $r_{\text {skin }} \simeq 0.21 \pm 0.02 \mathrm{fm}$ for ${ }^{208} \mathrm{~Pb}$. Some deliberations at this stage on the neutron skin $r_{\text {skin }}$ of ${ }^{208} \mathrm{~Pb}$ may be meaningful. A recent PREX experiment [47] reports a large central value of $0.33 \mathrm{fm}$ for $r_{\text {skin }}$ of ${ }^{208} \mathrm{~Pb}$ with very large error bars. This contradicts nearly all the calculated results of $r_{\text {skin }}$, which are comparatively much smaller. Fattoyev and Piekarewicz [48] devised a relativistic EoS that can accommodate such a large neutron skin, but then $e_{\text {sym }}\left(\rho_{0}\right)$ and $L_{0}$ become uncomfortably high. The larger the value of $r_{\text {skin }}$, the larger becomes the value of $L_{0}$. It is known that the larger is then the value of the neutron star radius [49]. A large neutron star radius seems to be incompatible with astrophysical data [50,51]; a very large value for the neutron skin of ${ }^{208} \mathrm{~Pb}$ is thus doubtful. Calculations by Brown [25] tend to disfavor a large neutron skin of ${ }^{208} \mathrm{~Pb}$. Nuclear ground state data for closed shell nuclei were fitted with a set of Skyrme interactions with constraints of fixed $r_{\text {skin }}$. The average deviation for binding energies was found to be similar for $r_{\text {skin }}=0.16$ and $0.20 \mathrm{fm}$, but increased by 0.1 to $0.3 \mathrm{MeV}$ for $r_{\text {skin }}=0.24 \mathrm{fm}$. A very recent experimental determination of the neutron skin thickness from coherent pion production [52] adds a new dimension to this issue: the extracted value of $r_{\text {skin }}$ for ${ }^{208} \mathrm{~Pb}$ is $r_{\text {skin }}=0.15 \pm 0.03 \mathrm{fm}$.

\section{Supranormal densities: Neutron stars}

Having come this far, we try to assess our EoS with reference to that extracted from experimental data at supranormal densities. This is done in Fig. 4. The upper panel displays the EoS (pressure density relation) of symmetric nuclear matter (SNM). The shaded red and yellow regions show the

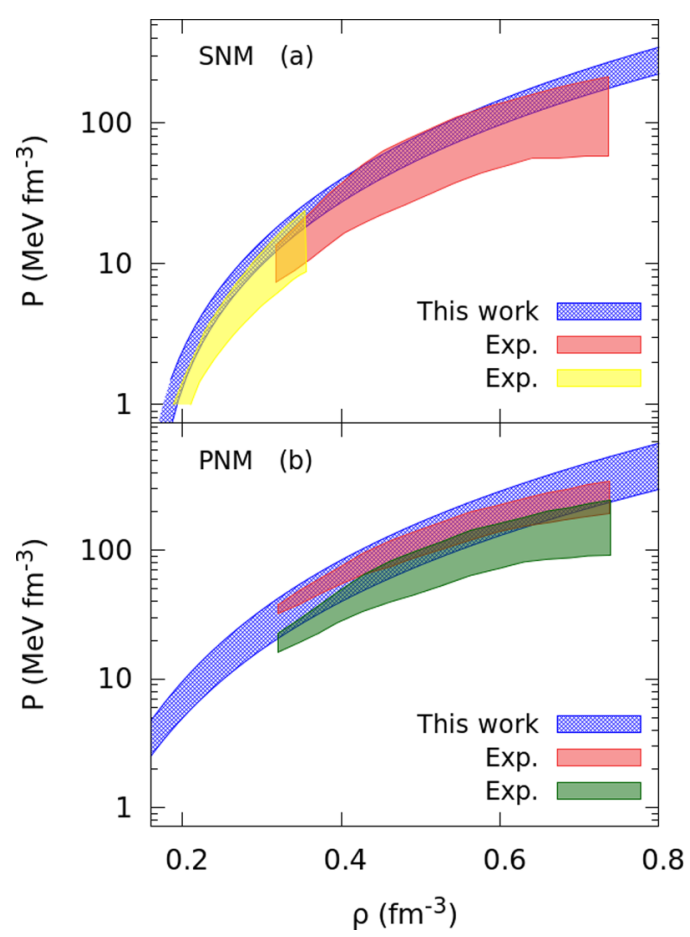

FIG. 4. (Color online) The EoS for symmetric nuclear matter (upper panel) and for pure neutron matter (lower panel). The red, yellow, and green shaded regions represent the experimental data taken from Refs. [53-56]. The blue shaded regions are the EoS obtained in this work. See text for details.

"experimental" EoS for SNM synthesized from collective flow data [53] and from data for kaon production [54,55], respectively; the blue shaded region shows ours. The EoS of pure neutron matter (PNM) has an additional repulsive component coming from the density dependence of symmetry energy. This part of the EoS is laced with uncertainty; it is model dependent. The lower panel shows the EoS of PNM. The shaded green region is the EoS of PNM where the density dependence of symmetry energy is modeled as soft; the red shaded region is the one where the said density dependence is modeled as stiff [56]. The blue shaded region displays the results obtained in the present work; it has an excellent overlap with the "experimental" EoS. Possible phase transitions to exotic phases such as hyperons, kaons, etc. at high densities soften the EoS somewhat; this is not taken into account in the present description.

For completeness, to gauge the applicability of the EoS to higher densities, we calculated the lower limit of the maximum mass of the neutron star $\left(M_{\max }^{\mathrm{NS}}\right)$ with this EoS, solving the general relativistic Tolman-Oppenheimer-Volkoff equation [57]. The EoS for the crust was taken from the Baym, Pethick, and Sutherland model [58]. The EoS for the core region was calculated under the assumption of a charge-neutral uniform plasma of neutrons, protons, electrons, and muons in $\beta$ equilibrium. The EoS is causal for $0 \leqslant \rho \leqslant 8.3 \rho_{0}$; the central density of the neutron star in our calculation never reaches beyond $\rho \sim 6 \rho_{0}$. Our value of $M_{\max }^{\mathrm{NS}}\left(=2.19 M_{\odot}\right)$ is consistent with the currently observed value of $1.97 \pm 0.04 M_{\odot}$ for 
the pulsar PSR J1614-2230 [59] and also the value of $2.01 \pm 0.04 M_{\odot}$ for the pulsar PSR J0348+0432 [60]. The presence of exotic degrees of freedom like hyperons in the core of the neutron star is known to pull down the value of $M_{\max }^{\mathrm{NS}}$ substantially [61]; however, in the relativistic mean-field (RMF) model, it is also seen that by increasing the strength of coupling of the hyperon to the vector mesons, the effect of hyperons on $M_{\max }^{\mathrm{NS}}$ can be much reduced [62]. Recently analyzing different models, Lattimer et. al $[50,63]$ constrained the value of the radius $R_{1.4}$ for a neutron star of mass $1.4 M_{\odot}$ to $12.1 \pm 1.1 \mathrm{~km}$ with $90 \%$ confidence level; our value of $R_{1.4}$ is $11.95 \pm 0.75 \mathrm{~km}$. Determination of neutron star radius is, however, not free from uncertainty. Assuming that the neutron star core is best described by a "normal matter" EoS, Guillot et al. [51] find, again in a $90 \%$ confidence level, that for astrophysically relevant masses $\left(M_{\mathrm{NS}} \geqslant 0.5 M_{\odot}\right)$, the neutron star radius is quasiconstant, $R_{\mathrm{NS}}=9.1_{-1.5}^{+1.3} \mathrm{~km}$.

\section{CONCLUSIONS}

To sum up, from consensus "empirical" inputs for values of some of the key nuclear parameters at saturation and subsaturation densities, we have constructed a Skyrme-type energy density functional for homogeneous nuclear matter. This is then employed to understand the density dependence of the nuclear symmetry energy and incompressibility and to predict values for the important nuclear parameters such as the symmetry slope parameter $L_{0}$, the symmetry incompressibility parameter $K_{\tau}$, and the incompressibility slope parameter $M(\rho)$. Separate estimates of these quantities have been given from different perspectives; sizable uncertainties remain there. The structural edifice for the energy density functional built on a few known input bulk parameters gives coherence to the evaluated values of the observables; their uncertainties can be constrained better provided the input bulk entities are known with better precision. The general agreement of our EoS with the "experimental" one at supranormal densities is interestingly striking. The near concordance of our calculated lower bound of the maximum mass of a neutron star with the experimental observation of a neutron star of mass $M_{\max }^{\mathrm{NS}} \sim$ $2 M_{\odot}$ is also very noticeable. Inclusion of exotic degrees of freedom in the interior of the star, however, softens the EoS and lowers the value of $M_{\mathrm{max}}^{\mathrm{NS}}$, and this needs further investigation.

\section{ACKNOWLEDGMENTS}

J.N.D. acknowledges support from the Department of Science and Technology, Government of India. G.C. would like to thankfully acknowledge the nice hospitality extended to him during his visit to SINP, when this work started. The authors gratefully acknowledge the assistance of Tanuja Agrawal in the preparation of the manuscript.
[1] P. Möller, W. D. Myers, H. Sagawa, and S. Yoshida, Phys. Rev Lett. 108, 052501 (2012).

[2] W. D. Myers and W. J. Swiatecki, Ann. Phys. (NY) 55, 395 (1969).

[3] W. D. Myers and W. J. Swiatecki, Nucl. Phys. A 336, 267 (1980).

[4] H. Jiang, G. J. Fu, Y. M. Zhao, and A. Arima, Phys. Rev. C 85, 024301 (2012).

[5] X. Fan, J. Dong, and W. Zuo, Phys. Rev. C 89, 017305 (2014).

[6] M. Liu, N. Wang, Z.-X. Li, and F.-S. Zhang, Phys. Rev. C 82, 064306 (2010).

[7] L. Trippa, G. Colò, and E. Vigezzi, Phys. Rev. C 77, 061304(R) (2008).

[8] A. Carbone, G. Colò, A. Bracco, L.-G. Cao, P. F. Bortignon, F. Camera, and O. Wieland, Phys. Rev. C 81, 041301(R) (2010).

[9] J. Dong, W. Zuo, J. Gu, and U. Lombardo, Phys. Rev. C 85, 034308 (2012).

[10] M. Centelles, X. Roca-Maza, X. Viñas, and M. Warda, Phys. Rev. Lett. 102, 122502 (2009).

[11] M. Warda, X. Viñas, X. Roca-Maza, and M. Centelles, Phys. Rev. C 80, 024316 (2009).

[12] L.-W. Chen, C. M. Ko, and B.-A. Li, Phys. Rev. C 72, 064309 (2005).

[13] B.-A. Li, L.-W. Chen, and C. M. Ko, Phys. Rep. 464, 113 (2008).

[14] M. A. Famiano et al., Phys. Rev. Lett. 97, 052701 (2006).

[15] D. V. Shetty, S. J. Yennello, and G. A. Souliotis, Phys. Rev. C 75, 034602 (2007).

[16] B. K. Agrawal, J. N. De, and S. K. Samaddar, Phys. Rev. Lett. 109, 262501 (2012).

[17] B. K. Agrawal, J. N. De, S. K. Samaddar, G. Colò, and A. Sulaksono, Phys. Rev. C 87, 051306(R) (2013).
[18] X. Roca-Maza, M. Brenna, G. Colò, M. Centelles, X. Viñas, B. K. Agrawal, N. Paar, D. Vretenar, and J. Piekarewicz, Phys. Rev. C 88, 024316 (2013).

[19] X. Roca-Maza, M. Brenna, B. K. Agrawal, P. F. Bortignon, G. Colò, L.-G. Cao, N. Paar, and D. Vretenar, Phys. Rev. C 87, 034301 (2013).

[20] Topical Issue on Nuclear Symmetry Energy, edited by B.-A. Li, A. Ramos, G. Verde, and I. Vidana, Special issue of Eur. Phys. J. A 50, 1 (2014).

[21] A. W. Steiner, M. Prakash, J. M. Lattimer, and P. J. Ellis, Phys. Rep. 411, 325 (2005).

[22] H.-T. Janka, K. Langanke, A. Marek, G. Martínez-Pinedo, and B. Müller, Phys. Rep. 442, 38 (2007).

[23] L. F. Roberts, G. Shen, V. Cirigliano, J. A. Pons, S. Reddy, and S. E. Woosley, Phys. Rev. Lett. 108, 061103 (2012).

[24] Z.-G. Xiao, G.-C. Yong, L.-W. Chen, B.-A. Li, M. Zhang, G.-Q. Xiao, and N. Xu, Eur. Phys. J. A 50, 37 (2014).

[25] B. A. Brown, Phys. Rev. Lett. 111, 232502 (2013).

[26] E. Khan, J. Margueron, and I. Vidaña, Phys. Rev. Lett. 109, 092501 (2012).

[27] S. Shlomo, V. M. Kolomietz, and G. Colò, Eur. Phys. J. A 30, 23 (2006).

[28] B. K. Agrawal, S. Shlomo, and V. K. Au, Phys. Rev. C 72, 014310 (2005).

[29] M. Dutra, O. Lourenço, J. S. Sá Martins, A. Delfino, J. R. Stone, and P. D. Stevenson, Phys. Rev. C 85, 035201 (2012).

[30] B. D. Serot and J. D. Walecka, Adv. Nucl. Phys. 16, 1 (1986).

[31] B. D. Serot and J. D. Walecka, Int. J. Mod. Phys. E 6, 515 (1997).

[32] M. Brack, C. Guet, and H.-B. Hakansson, Phys. Rep. 123, 275 (1985). 
[33] B. Cochet, K. Bennaceur, J. Meyer, P. Bonche, and T. Duguet, Int. J. Mod. Phy. E 13, 187 (2004).

[34] L.-W. Chen, B.-J. Cai, C. M. Ko, B.-A. Li, C. Shen, and J. Xu, Phys. Rev. C 80, 014322 (2009).

[35] C. Constantinou, B. Muccioli, M. Prakash, and J. M. Lattimer, Phys. Rev. C 89, 065802 (2014).

[36] C.-H. Lee, T. T. S. Kuo, G. Q. Li, and G. E. Brown, Phys. Rev. C 57, 3488 (1998).

[37] I. Vidaña and I. Bombaci, Phys. Rev. C 66, 045801 (2002).

[38] G. Colò, U.Garg, and H. Sagawa, Euro. Phys. J A 50, 26 (2014).

[39] I. Vidaña, C. Providência, A. Polls, and A. Rios, Phys. Rev. C 80, 045806 (2009).

[40] T. Li et al., Phys. Rev. Lett. 99, 162503 (2007).

[41] J. Piekarewicz and M. Centelles, Phys. Rev. C 79, 054311 (2009).

[42] J. Piekarewicz, J. Phys. G 37, 064038 (2010).

[43] J. M. Pearson, N. Chamel, and S. Goriely, Phys. Rev. C 82, 037301 (2010).

[44] M. M. Majumdar, S. K. Samaddar, N. Rudra, and J. N. De, Phys. Rev. C 49, 541 (1994).

[45] G. Arfken and H. Weber, Mathematical Methods for Physicists (Academic Press, Waltham, MA, 2005).

[46] C. Ducoin, J. Margueron, C. Providência, and I. Vidaña, Phys. Rev. C 83, 045810 (2011).

[47] S. Abrahamyan et al., Phys. Rev. Lett. 108, 112502 (2012).

[48] F. J. Fattoyev and J. Piekarewicz, Phys. Rev. Lett. 111, 162501 (2013).
[49] C. J. Horowitz and J. Piekarewicz, Phys. Rev. C 64, 062802(R) (2001).

[50] J. M. Lattimer and A. W. Steiner, Euro. Phys. J 50, 40 (2014).

[51] S. Guillot, M. Servillat, N. Webb, and R. Rutledge, Astrophys. J 772, 7 (2013).

[52] C. M. Tarbert, D. P. Watts, D. I. Glazier, P. Aguar et al., Phys. Rev. Lett. 112, 242502 (2014).

[53] P. Danielewicz, W. G. Lynch, and R. Lacey, Science 298, 1592 (2002).

[54] C. Fuchs, Prog. Part. Nucl. Phys. 56, 1 (2006).

[55] A. F. Fantina, N. Chamel, J. M. Pearson, and S. Goriely, EPJ Web Conf. 66, 07005 (2014).

[56] M. Prakash, T. L. Ainsworth, and J. M. Lattimer, Phys. Rev. Lett. 61, 2518 (1988).

[57] S. Weinberg, Gravitation and Cosmology (Wiley, New York, 1972).

[58] G. Baym, C. Pethick, and P. Sutherland, Astrophys. J. 170, 299 (1971).

[59] P. B. Demorest, T. Pennucci, S. M. Ransom, M. S. E. Roberts, and J. W. T. Hessels, Nature (London) 467, 1081 (2010).

[60] J. Antoniadis et al., Science 340, 448 (2013).

[61] H. J. Schulze and T. Rijken, Phys. Rev. C 84, 035801 (2011).

[62] S. Weissenborn, D. Chatterjee, and J. Schaffner-Bielich, Phys. Rev. C 85, 065802 (2012).

[63] J. M. Lattimer and Y. Lim, Astrophys. J. 771, 51 (2013). 